\title{
Some New Properties of the Eshelby Stress Tensor
}

\author{
Erwan Verron, Malik Aït-Bachir and Philippe Castaing
}

\begin{abstract}
The Eshelby stress tensor is known to be an appropriate Continuum Mechanics quantity to capture singularities. Nevertheless, even if its use in the calculation of configurational forces is well-established, its peculiar properties were investigated only recently. Here, some new properties of this tensor are studied. In this way, it is assumed that the evolution of microscopic defects in the material can be predicted at the macroscopic scale by examining the components of the Eshelby stress tensor. More precisely, considering that defects can be modeled by material surfaces oriented in all possible directions and assuming that they are able to evolve in every possible directions, it is shown that the maximum amount of energy which can be released by defects evolution is partially contained in the tensor. In the special case of hyperelasticity, the corresponding optimization problem is established and solved for both isotropic and anisotropic materials.
\end{abstract}

\section{Introduction}

The theory of Configurational Mechanics was introduced by Eshelby in [4] when he proposed the concept of energy-momentum tensor and configurational forces in continuum mechanics of solids by studying the driving force of a moving defect. Twenty years later, both Eshelby [5] and Chadwick [2] extended the previous

\footnotetext{
Erwan Verron

Institut de Recherche en Génie Civil et Mécanique, UMR CNRS 6183, École Centrale de Nantes, BP 92101, 44321 Nantes Cedex 3, France; e-mail: erwan.verron@ec-nantes.fr

Malik Aït-Bachir

Institut de Recherche en Génie Civil et Mécanique, UMR CNRS 6183, École Centrale de Nantes, BP 92101, 44321 Nantes Cedex 3, France; e-mail: malik.ait-bachir@ec-nantes.fr

Philippe Castaing

Centre Technique des Industries Mécaniques, 74 route de la Jonelière, BP 82617, 44326 Nantes

Cedex 3, France; e-mail: philippe.castaing@ @etim.fr
} 
theory to finite strain. More recently some authors revisited these studies and established the general framework of Configurational Mechanics also designated as the Eshelbian Mechanics and Mechanics in the Material Space [6,9,11,12].

In the majority of studies involving Configurational Mechanics, only configurational forces are investigated through the calculation of path-independent integrals around inhomogeneities, because these forces are recognized as the driving forces of defect evolution. So, the Eshelby stress tensor only appears in the definition of surface tractions, i.e. after contraction with the outward normal of the contour. Most of these works focus on Fracture Mechanics (see for example [16] and the references herein). Moreover, as proposed in [10], other problems can be analyzed with the help of the Eshelbian framework: dislocations in metal, movement of interfaces in two-phase bodies, etc. Opposite to the case of configurational forces, only few studies are concerned with the peculiar properties of the Eshelby stress tensor. As an example, for the linear theory, the physical significance of the Cartesian components of this tensor were identified only recently by Kienzler and Herrmann [8]. More recently, Verron and co-workers proposed to use some components of the Eshelby stress tensor to predict fatigue damage in elastomers [1, 17, 18].

By generalizing and rationalizing the derivation proposed in [17], the present paper will examine some properties of the Eshelby stress tensor. In this way, the evolution of the microstructure of a given material will be studied by considering the evolution of oriented material surfaces under loading. The approach will exhibit the relevance of the polar decomposition of the tensor and define the significance of this decomposition with regards to microstructural evolution. Finally, this work will be illustrated by considering the extension of a transversely isotropic hyperelastic strip.

\section{Evolution of Microstructural Defects by Considering the Eshelby Stress Tensor}

\subsection{Formulation of the Problem}

Consider a body defined by its reference configuration $\left(\mathcal{C}_{R}\right)$, i.e. a set of particles in the material manifold $\mathcal{M}^{3}$; it is depicted in Figure 1. One particle $P$ of this set is located at $\mathbf{X}$ in the physical space $\varepsilon^{3}$. Under mechanical loading, the body deforms and occupies a time sequence of physical configurations (in $\left.\mathcal{E}^{3}\right)$. Let $(\mathcal{C})$ be the body configuration at time $t$ defined by the mapping $\mathbf{x}(\mathbf{X}, t)$ and by its gradient $\mathbf{F}(\mathbf{X}, t)$. As shown in Fig. 1, when loading is removed the body will, in general, occupy a new stress-free configuration $\left(\mathcal{C}_{R}^{\prime}\right)$ defined by the motion gradient $\mathbf{f}(\mathbf{x}, t)$. Both configurations $\left(\mathcal{C}_{R}\right)$ and $\left(\mathcal{C}_{R}^{\prime}\right)$ represent natural configurations of the body [14]. The physical reasons for a body to possess different natural configurations can be very diverse. Indeed, it is the consequence of structural rearrangements at the microscopic scale, e.g. movement of dislocations, cavitation, cleavage fracture, 
Fig. 1 Deformation of a body: macroscopic scale.

Fig. 2 Idealized microstructural change in the RVE

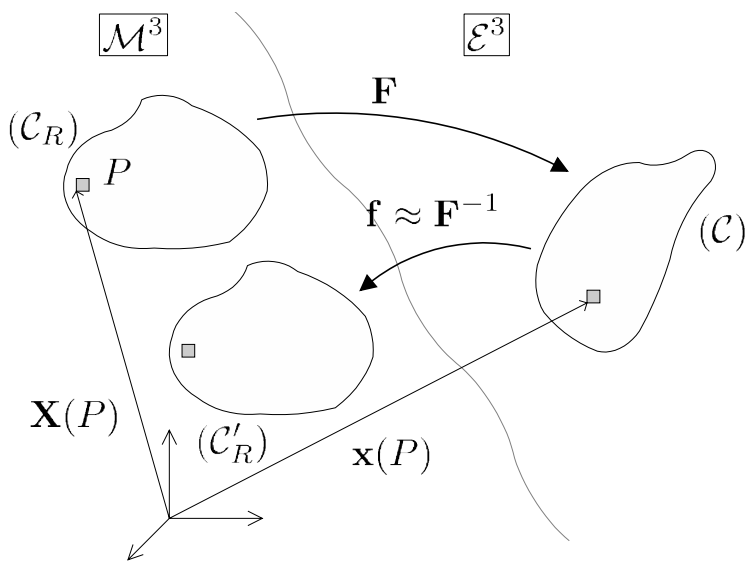

$$
\left(\mathcal{C}_{R}\right)
$$

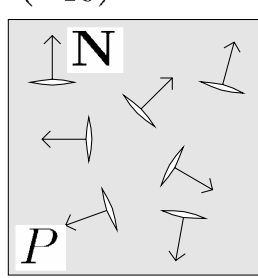

$(\mathcal{C})$

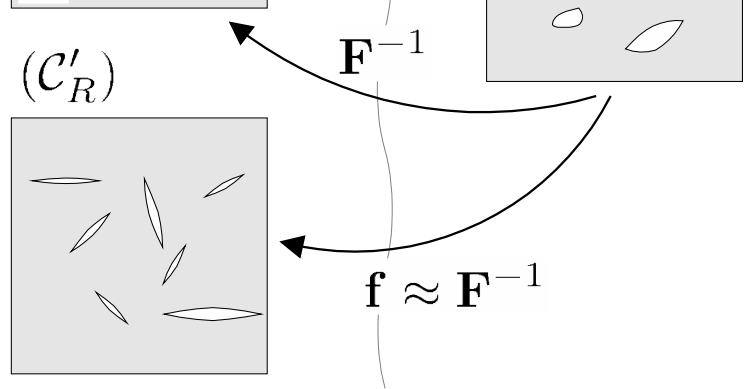

which manifests in various ways at the mesoscopic scale, e.g. plasticity, damage; or at the macroscopic scale, e.g. crack growth. If the material is perfectly elastic, $\mathbf{f}=\mathbf{F}^{-1}$ and the natural configurations $\left(\mathcal{C}_{R}\right)$ and $\left(\mathcal{C}_{R}^{\prime}\right)$ are identical. Nevertheless, if irreversible microstructural changes take place during the motion, the gradient $\mathbf{f}$ can be considered close, but not equal, to $\mathbf{F}^{-1}$.

In the latter case, this slight change of configuration is the macroscopic counterpart of a microstructural evolution. In order to illustrate this change, the Representary Volume Element (RVE) which is phenomenologically embedded in the particle $P$ should be considered. The following idealized representation of the RVE is adopted: it contains both bulk material and various defects which are schematized by oriented material surfaces as shown in Figure 2. These surfaces, which can repres- 
Fig. 3 Evolution of the defects modeled by oriented material surfaces.

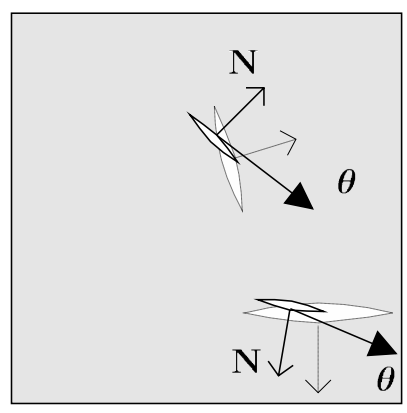

ent for example microscopic cracks, are defined by their normal vector $\mathbf{N}$ and it is assumed that their orientations are isotropically distributed. Under motion, the size and shape of the RVE (see the grey square in Figure 2) do not change: the microstructural rearrangement does not change the definition of the RVE, i.e. at the Continuum Mechanics scale, the motion is reversible. Nevertheless, the defects deform reversibly or irreversibly, and in the latter case the microstructural rearrangement leads to the definition of the new reference configuration $\left(\mathcal{C}_{R}^{\prime}\right)$ in Figures 1 and 2.

As advocated in $[3,13]$, the Eshelby stress tensor $\boldsymbol{\Sigma}$ is the driving force which governs local structural rearrangement. This tensor is defined by

$$
\boldsymbol{\Sigma}=W \mathbf{I}-\mathbf{F}^{t} \mathbf{P}
$$

where $W$ is the strain energy density per unit of undeformed volume which a priori depends on both $\mathbf{F}$ and $\mathbf{X}, \mathbf{P}$ is the Piola-Kirchhoff stress tensor and ${ }^{t}$ denotes the transposition. To quantify the energy change involved during the rearrangement, we consider that a given material surface (defined by its unit normal vector $\mathbf{N}$ ) is subjected to a material unit translation $\boldsymbol{\theta}$ between the natural configurations $\left(\mathcal{C}_{R}\right)$ and $\left(\mathcal{C}_{R}^{\prime}\right)$ as shown in Figure 3. In 1997, Kienzler and Herrmann identified the physical significance of the components of the Eshelby stress tensor in the linear context [8]: "[the $i j$-component] of the Eshelby tensor is the change in the total energy density at a point of an elastic continuum due to a material unit translation in $x_{j}$ direction of a unit surface with normal in $x_{i}$-direction". Extending this definition to finite strain and to arbitrary direction, it can be established that the scalar $\boldsymbol{\theta} \cdot \boldsymbol{\Sigma} \mathbf{N}$ represents the change of energy due to the evolution of the material surface defined by the unit normal vector $\mathbf{N}$ and the material unit translation $\boldsymbol{\theta}$ between the natural configurations $\left(\mathcal{C}_{R}\right)$ and $\left(\mathcal{C}_{R}^{\prime}\right)$.

To go further in the derivation, a strong assumption is adopted: it is considered that the microstructural change is only due to the evolution of only one set of surfaces (only one orientation of defects, i.e. only one vector $\mathbf{N}$ ) in only one material translation (only one vector $\boldsymbol{\theta}$ ) such that the body reduces as much as possible its total energy. Recalling that energy changes are defined positively in $\Sigma$, the problem reduces to the following constraint optimization problem:

$$
\max _{\mathbf{N}, \boldsymbol{\theta}} \theta \cdot(-\mathbf{\Sigma}) \mathbf{N}
$$


with two equality constraints

$$
\|\mathbf{N}\|=1, \quad\|\boldsymbol{\theta}\|=1,
$$

and two inequality constraints

$$
\mathbf{N} \cdot \boldsymbol{\theta} \geq 0, \quad \boldsymbol{\theta} \cdot(-\boldsymbol{\Sigma}) \mathbf{N} \geq 0 .
$$

The two equality constraints Eq. (3) specify that both the normal vector to the material surface and the material translation vector are unit vectors. The inequality constraints Eq. (4) express that the microscopic defects can only open: Eq. (4) 1 ensures that $\mathbf{N}$ and $\boldsymbol{\theta}$ are in the same semi-plan, Eq. (4) 2 ensures that energy can only decrease due to defects evolution. Finally, the scalar measure of the damage $\Sigma^{*}$ can be chosen as the maximum of the function $\theta \cdot(-\boldsymbol{\Sigma}) \mathbf{N}$.

Remark. The quantity $\Sigma^{*}$ which solves the optimization problem represents the energy involved in the evolution of the oriented material surface (with normal $\mathbf{N}$ ). The assumptions required to use the Eshelby stress tensor in this context are: (i) the size of defects is very small compared with the size of the RVE, and (ii) the density of defects is also very small, i.e. defects do not interact. It means that this approach is able to localize the damage in the material, but can not be used to calculate the energy release rate of defects because such a quantity obviously includes the size of defects.

\subsection{Mathematical Solution}

\subsubsection{Special Case of an Isotropic Elastic Material}

As shown in [3], the Eshelby stress tensor satisfies the following symmetry condition:

$$
\mathbf{\Sigma} \mathbf{C}=\mathbf{C} \boldsymbol{\Sigma}^{t},
$$

where $\mathbf{C}$ is the right Cauchy-Green strain tensor. Moreover, recalling that $\boldsymbol{\Sigma}$ can be written in terms of $\mathbf{C}$ and of the second Piola-Kirchhoff stress tensor $\mathbf{S}$,

$$
\boldsymbol{\Sigma}=W \mathbf{I}-\mathbf{C S}
$$

and noting that in the special case of isotropic elasticity $\mathbf{C}$ and $\mathbf{S}$ are coaxial and commute, the Eshelby stress tensor is symmetric.

So, $\boldsymbol{\Sigma}$ being a symmetric tensor, it possesses three real eigenvalues denoted $\left(\Sigma_{i}\right)_{i=1,3}$ and their corresponding eigenvectors $\left(\mathbf{V}_{i}\right)_{i=1,3}$ which are orthogonal one to each other. In this case, the solution of the optimization problem reduces to:

$$
\Sigma^{*}=\left|\min \left(\left(\Sigma_{i}\right)_{i=1,2,3}, 0\right)\right|
$$


and if $\Sigma^{*} \neq 0$,

$$
\mathbf{N}=\boldsymbol{\theta}=\mathbf{V}^{*},
$$

which is the eigenvector associated with $-\Sigma^{*}$. In this case, the defects with normal $\mathbf{V}^{*}$ will grow by extending in a plane orthogonal to $\mathbf{V}^{*}$.

\subsubsection{General Case}

More generally, the Eshelby stress tensor is not symmetric, it only satisfies Eq. (5). In order to solve the optimization problem, we consider the right polar decomposition of $\Sigma$ :

$$
\Sigma=\Gamma \Upsilon
$$

where $\boldsymbol{\Gamma}$ is an orthogonal tensor (a reflection or a rotation tensor depending on the sign of its determinant) and $\boldsymbol{\Upsilon}$ is a positive definite symmetric tensor (when $\boldsymbol{\Sigma}$ is invertible). These tensors are defined by

$$
\Upsilon=\sqrt{\Sigma^{t} \Sigma} \text { and } \Gamma=\Sigma \Upsilon^{-1} .
$$

In this case the solution vectors are

$$
\mathbf{N}=\mathbf{V}^{*} \text { and } \boldsymbol{\theta}=\boldsymbol{\Gamma} \mathbf{V}^{*}
$$

where $\mathbf{V}^{*}$ is the eigenvector associated with the largest eigenvalue of $\boldsymbol{\Upsilon}$. If these vectors are not in the same semi-plan, i.e. if they do not satisfy Eq. (4) ${ }_{1}$, the solution of the whole optimization problem (with constraints) Eqs. (2-4) is equal to 0 similarly to Eq. (7). Otherwise, the maximum of the function $\boldsymbol{\theta} \cdot(-\boldsymbol{\Sigma}) \mathbf{N}$ is the largest eigenvalue of $\Upsilon$ :

$$
\Sigma^{*}=\max \left(\left(\Upsilon_{i}\right)_{i=1,3}\right) .
$$

In this case, the defects with normal $\mathbf{V}^{*}$ will grow by extending (and being distorted) in a plane of normal $\Gamma \mathbf{V}^{*}$.

\section{Example}

First, the case in which the Eshelby stress tensor is symmetric and which corresponds to isotropic elasticity was recently examined in [17]. Authors applied this result to the problem of fatigue loading of rubber materials. Using Eq. (7), they derived a new predictor for rubber fatigue and they demonstrated its ability to reproduce multiaxial loading conditions and to predict macroscopic fatigue crack orientation.

So, the following example will focus on the second result derived in Section 2.2.2, Eqs (11-12). We consider the simple problem of uniaxial extension of a transversely isotropic hyperelastic strip. More precisely, the problem consists in extending a rubber-like thin strip reinforced with long fibers oriented in a given 
Fig. 4 Extension of a reinforced fiber elastic strip.

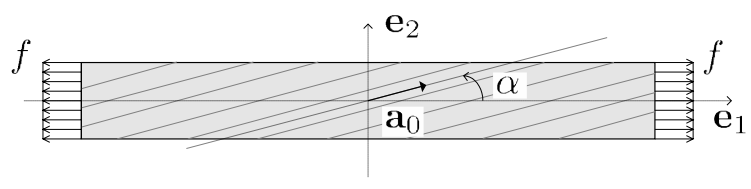

direction $\mathbf{a}_{0}$, as shown in Figure 4 . The angle between the fiber direction, i.e. $\mathbf{a}_{0}$, and the loading direction, i.e. $\mathbf{e}_{1}$, is denoted $\alpha$. Following the work of Spencer [15], the strain energy density per unit of undeformed volume for a homogeneous transversely isotropic material $W$ can be written as a function of five invariants: the three classical isotropic invariants

$$
I_{1}=\operatorname{tr} \mathbf{C}, \quad I_{2}=\frac{1}{2}\left[(\operatorname{tr} \mathbf{C})^{2}-\operatorname{tr} \mathbf{C}^{2}\right], \quad I_{3}=\operatorname{det} \mathbf{C},
$$

and two additional invariants

$$
I_{4}=\operatorname{tr}(\mathbf{C}: \mathbf{A}), \quad I_{5}=\operatorname{tr}\left(\mathbf{C}^{2}: \mathbf{A}\right),
$$

where $\mathbf{A}$ is the orientation tensor $\mathbf{a}_{0} \otimes \mathbf{a}_{0}$. Here, the simplest transversely isotropic rubber-like constitutive equation is adopted: the material is considered incompressible $\left(I_{3}=1\right)$ and the strain energy density is assumed to only depends on $I_{1}$ and $I_{4}$

$$
W\left(\mathbf{C}, \mathbf{a}_{0}\right)=C_{1}\left(I_{1}-3\right)+C_{4}\left(I_{4}-1\right)^{2},
$$

where the two material parameters were set to $C_{1}=1$ and $C_{4}=2$ in order to emphasize the effect of fibers. In the special cases for which the loading direction is parallel $\left(\alpha=0^{\circ}\right)$ or perpendicular $\left(\alpha=90^{\circ}\right)$ to the fiber direction, a given extension can be prescribed and the problem can be solved analytically (see for example [7]). Nevertheless, for other fiber orientations the finite element method should be considered; here we used the software COMSOL Multiphysics. Computations are performed by considering only half of the strip (symmetry with respect to the $x_{1}=0$-axis), the plane stress assumption is adopted (the incompressibility constraint leads to a change in thickness of the strip) and the strip is extended by prescribing the force $f$ in order to obtain an uniform deformation gradient in the whole strip.

Practically, for a given extension ratio $\lambda$, simulations with prescribed force $f$ are conducted to determine the value of the force such that $F_{11}=\lambda$. To show quantitative results we plot the evolution of the directions of $\mathbf{V}^{*}$ (normal vector to the critical material plane) and $\boldsymbol{\Gamma} \mathbf{V}^{*}$ (material direction of evolution) with respect to the fiber directions in Figure 5. It is to note that for both $\alpha=0^{\circ}$ and $\alpha=90^{\circ}$, even if the material is not isotropic, the deformation gradient is diagonal and then the Eshelby stress tensor is symmetric. Thus the problem reduces to the special case studied in Section 2.2.1; the normal vector to the material surfaces and the normal to the plane in which they will grow are identical. In other cases, they are different. 

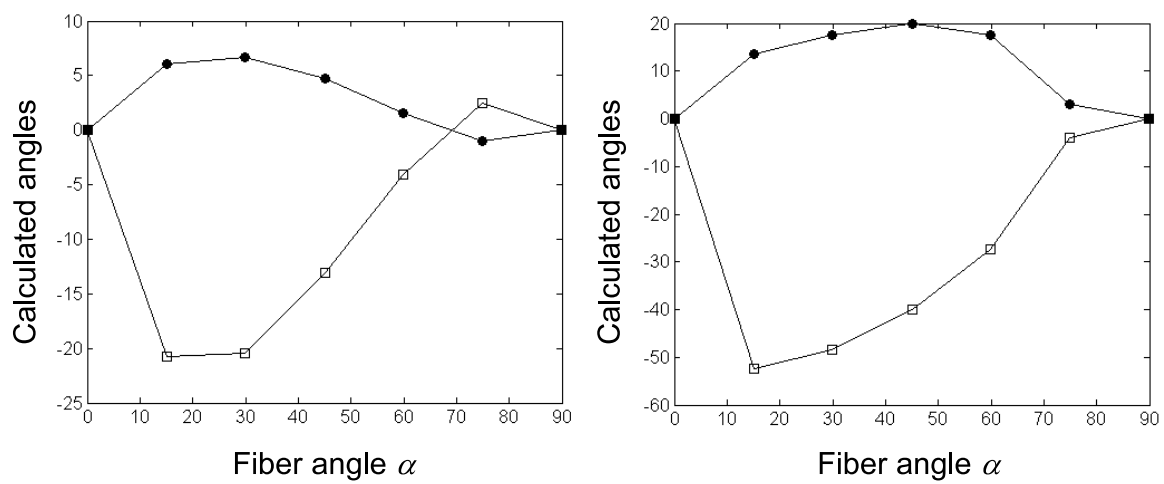

Fig. 5 (•) Orientation of the critical material plane which could release the maximum of en-

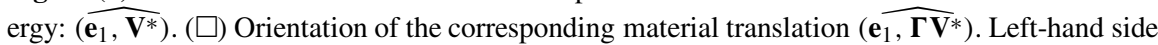
graph: $\lambda=2$; right-hand side graph: $\lambda=4$.

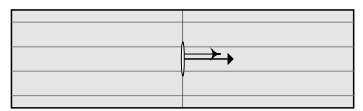

$(a)$

Fig. $6 \lambda=4$-extension of a transversaly isotropic hyperelastic strip: (a) $\alpha=0^{\circ}$, (b) $\alpha=30^{\circ}$, (c) $\alpha=60^{\circ}$ and (d) $\alpha=90^{\circ}$.

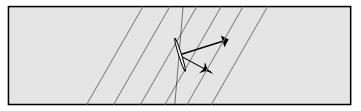

(c)

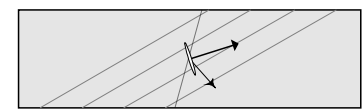

(b)

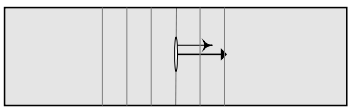

$(d)$

Finally, Figure 6 shows some qualitative results obtained for four fiber orientations. This figure illustrates with simple sketches four particular quantitative results given in Figure 5: for $\lambda=4$, it exhibits the orientation of the critical material surfaces $\left(\mathbf{V}^{*}\right)$ and the direction of the material translation $\left(\boldsymbol{\Gamma} \mathbf{V}^{*}\right)$ for four fiber orientation angles.

\section{Discussion}

The present paper is only a second step towards the understanding of the physical significance of the Eshelby stress tensor components, after the one of Kienzler and Herrmann. The relevance of the polar decomposition of the Eshelby stress tensor was demonstrated by considering that this tensor is the driving force of microstructural evolution. For an idealized RVE, it was shown that the "pure material stress tensor" $\Upsilon$ can be considered as a measure of the microstructural damage and of the direction of defects that will grow, and also that the "rotational material stress tensor" $\boldsymbol{\Gamma}$ contains the way these defects will evolve. 


\section{References}

1. Andriyana, A. and Verron, E., Prediction of fatigue life improvement in natural rubber using configurational stress. Int. J. Solids Struct. 44, 2007, 2079-2092.

2. Chadwick, P., Applications of an energy-momentum tensor in non-linear elastostatics. J. Elast. 5, 1975, 249-258.

3. Epstein, M. and Maugin, G.A., (1990) The energy-momentum tensor and material uniformity in finite elasticity. Acta Mech. 83, 1990, 127-133.

4. Eshelby, J.D., The force on an elastic singularity. Phil. Trans. R. Soc. Lond. A 244, 1951, $87-112$.

5. Eshelby, J.D., The elastic energy-momentum tensor. J. Elast. 5, 1975, 321-335.

6. Gurtin, M.E., Configurational Forces as Basic Concept of Continuum Physics. Springer, Berlin, 2000.

7. Horgan, C.O. and Saccomandi, G., A new constitutive theory for fiber-reinforced incompressible nonlinearly elastic solids. J. Mech. Phys. Solids 53, 2005, 1985-2015.

8. Kienzler, R. and Herrmann, G., On the properties of the Eshelby tensor. Acta Mech. 125, 1997, 73-91.

9. Kienzler, R. and Herrmann, G., Mechanics in Material Space. Springer, Berlin, 2000.

10. Kolling, S., Mueller, R. and Gross, D., A computational concept for the kinetics of defects in anisotropic materials. Comput. Mat. Sci. 26, 2003, 87-94.

11. Maugin, G.A., Material Inhomogeneities in Elasticity. Chapman and Hall, London, 1993.

12. Maugin, G.A., Material forces: Concepts and applications. Appl. Mech. Rev. 48, 1995, 213245.

13. Maugin, G.A., Material mechanics of materials. Theor. Appl. Mech., 27, 2002, 1-12.

14. Rajagopal, K.R. and Srinivasa, A.R., On the role of the Eshelby energy-momentum tensor in materials with multiple natural configurations. Math. Mech. Solids 10, 2005, 3-24.

15. Spencer, A.J.M., Continuum Theory of the Mechanics of Fibre-reinforced Composite. Springer-Verlag, Berlin, 1984.

16. Steinmann, P., Application of material forces to hyperelastostatic fracture mechanics. I. Continuum mechanical setting. Int. J. Solids Struct. 37, 2000, 7371-7391.

17. Verron, E. and Andriyana, A., Definition of a new predictor for multiaxial fatigue crack nucleation in rubber. J. Mech. Phys. Solids 56, 2008, 417-443.

18. Verron, E., Le Cam, J.-B. and Gornet, L., A multiaxial criterion for crack nucleation in rubber. Mech. Res. Commun. 33, 2006, 493-498. 